\title{
LIDERES RESILIENTES Y MANAGEMENT DEL EMPORIO COMERCIAL DE GAMARRA DEL DISTRITO DE LA VICTORIA. RESILIENT LEADERS AND MANAGEMENT OF GAMARRA COMMERCIAL EMPORIUM, "LA VICTORIA" DISTRICT.
}

\author{
Autores: Mg. Florez Garmendia Pedro y Mg. Farfán Flores Moisés
}

\section{RESUMEN}

En un mundo lleno de cambios, transformaciones e incertidumbre económica y financiera, ¿por qué algunas organizaciones fracasan y otras llegan a recuperarse con, incluso mejores resultados que antes.? Más que centrarse en las causas que generan esta situación, la resiliencia organizacional y en especial los lideres resilientes, crean ventajas competitivas sobre otras empresas, al saber administrar-management- las adversidades internas y externas, enfrentándolas, sobreponiéndolas y aprendiendo de ellas para fortalecerse.

Las empresas que desarrollan la resiliencia son capaces de seguir proyectándose en el futuro a pesar de tener delante un entorno difícil y complicado que la crisis económica les está imponiendo, pero hay empresas que presentan un desempeño superior a otras, que no sólo subsisten sino que aún mejoran sus resultados.

¿En qué se diferencian estas organizaciones de las que en su gran mayoría sufren los embates perniciosos de las situaciones críticas?

Las empresas resilientes transmiten optimismo, resisten a la adversidad, saben adaptarse al entorno que les rodea, aprenden y desaprenden sin limitaciones, son capaces de mantener la confianza en lo que hacen, en su proyecto de empresa. El estudio, Lideres resilientes y management del emporio comercial de Gamarra, estuvo enmarcado dentro del nivel de investigación tipo descriptivo y explicativo con un estudio cualitativo y un diseño no experimental y se buscó determinar, como los lideres resilientes influyen en los procesos del management del emporio comercial de Gamarra del Distrito de la Victoria. La población estuvo conformada con una población de 364 comerciantes y una muestra 48 confeccionistas y textiles .Se privilegia la teoría de Wolin \& Wolin (1993) que privilegia los argumentos de la resiliencia.

Palabras claves : Lideres resilientes, Empresas resilientes, Management, Aprendizaje organizacional.

\section{SUMMARY}

In a world full of changes, transformations and economic and financial uncertainty, why some organizations fail and others achieve to recover with even better results than before? Rather than focusing on the causes of this situation, especially organizational resilience and resilient leaders, create competitive advantages over other companies, to know how to manage - management - internal and external adversities facing them, endure them and learning from them to become stronger.

Companies that develop resilience are able to continue projecting into the future despite having ahead a difficult and challenging environment that the economic crisis is imposing 
them, but there are companies that have a performance superior to others, which not only persist but even improve their results.

What these organizations that mostly suffer the pernicious attacks of critical situations differ?

Resilient companies transmit optimism, resisting adversity, know how to adapt to the environment around them, learn and unlearn without limitation, they are able to maintain confidence in what they do in their business project. The study, Resilient Leaders and management of the commercial emporium of Gamarra, was framed within the level of descriptive and explanatory type research with a qualitative study and a non-experimental design and sought to determine, as the resilient leaders influence the processes of management of the Emporium Gamarra commercial district of Victoria. The population consisted of a population of 364 dealers and a sample 48 garment manufacturers and textile .The theory favors Wolin \& Wolin (1993) that favors the arguments of resilience.

Keywords : Leaders resilient, resilient Business, Management, Organizational Learning.

\section{INTRODUCCIÓN}

La resiliencia es la capacidad que tienen las personas o las empresas para afrontar momentos de adversidad y adaptarse rápidamente a los cambios, que como consecuencia se dan dentro de esos períodos de inestabilidad. Desde la perspectiva empresarial sucede exactamente lo mismo; las crisis empresariales o los desequilibrios económicos y competitivos que se dan en el medio empresarial, pueden ser afrontados solamente a través de la utilización de la creatividad y la innovación. Es importante resaltar que no se trata de sobrevivir y tampoco de resistir; va más allá, se trata de aprovechar positivamente la experiencia estresante, para obtener un beneficio que no estaba planeado, pero que en el momento se convierte en una oportunidad para crear soluciones de beneficio individual (persona o empresa), que posteriormente al ser replicados se pueden llegar a convertir en estándares de desempeño colectivo. Hace varias décadas, la intersección de los jirones Gamarra e Hipólito Unanue, en el Distrito La Victoria, era un punto sin importancia en una zona abarrotada de pequeños restaurantes, bares, hostales y prostitución. Hoy se ha convertido en una de las zonas más cotizadas de este emporio comercial de confecciones, con alquileres mensuales, que superan los $\$ 10.000$ (dólares americanos) por un local de $60 \mathrm{~m} 2$, los fundadores de Gamarra, jamás imaginaron este crecimiento.

Pese a que no se disponen de cifras oficiales,- flujo y reflujo poblacional, -se estima que existe 170 galerías agrupadas en 20 manzanas, que albergan - aproximadamente17.000 locales manejados por 10.000 empresarios, que emplean más de 50.000 trabajadores en sus diferentes modalidades de servicios, contratos, destajo, etc. Las

encuestadoras como DATUM, y CUANTO, estiman que Gamarra, recibe cerca de 60.000 visitantes al día. 
Desde, que entro en vigencia el Tratado de Libre Comercio (TLC) con China en el 2010, la industria textil y confecciones peruana, se perjudico con el ingreso de prendas subvaluadas al mercado. A causa de ello, se estima, que este año se pierdan uno 30 mil empleos en zona productiva y comercial de Lima. (Fuente: Cámara de Comercio de Lima, Adex, SUNAT.)

El presidente del Comité de confecciones de la Sociedad de Industrias (SIN) Felipe James Callao, ratifica esta información al reconocer que las confecciones "pasan por momentos críticos " sumado a que las exportaciones están cayendo, un $20 \%$ frente al periodo 2012, además del $80 \%$ de las prensas son importaciones Chinas . (Diario La República 24/08/13). Añadido a estos problemas comerciales, nacionales e internacionales, el Distrito de La Victoria al margen de su historia y tradición tiene sectores, como parques, avenidas y calles, que se han convertido en zonas de eminente peligro, por la existencia de personas indeseables, pandillas, y otros establecimientos de dudosa condición, lo que compromete la integridad personal de empresarios, clientes y turistas, que concurren al emporio de Gamarra.

Ante esta difícil y compleja situación nos preguntamos ¿Cómo, los líderes resilientes influyen en los procesos del management del emporio comercial de Gamarra del distrito de la Victoria?. Además de describir las características de los lideres resilientes en el emporio Gamarra e Identificar los procesos de management en los sectores textiles y confecciones

La importancia y justificación del artículo radica porque pretende descubrir las estrategias de cómo los líderes resilientes revierten situaciones críticas, sistematizar y mejorar planes de contingencia para llegar a ser competitivos, focalizar los esfuerzos en los traumáticas. León (2015), trata de demostrar como las empresas resilientes son las que están preparadas para vencer los retos que se le presentan en las diferentes áreas del mercado globalizado en que realizan sus actividades, mejorando su eficiencia; manteniendo sus mercados; llegando a la conclusión que las empresas pueden aumentar su eficiencia en un $20 \%$ aplicando este innovador concepto.

\section{Sistema de Hipótesis}

$\mathrm{Hi}$ : Como los lideres resilientes influyen, significativamente, en los procesos del management del emporio comercial de Gamarra del Distrito de la Victoria.

Ho: Las mujeres presentan un perfil más satisfactorio en relación a los varones, en función al liderazgo y el management.

\section{MATERIALES Y MÉTODOS}

El tipo de diseño que se aplicó, fue el No experimental, pues dada las características del estudio, no existió ninguna manipulación deliberada de variables, por el contrario se utilizó la técnica de la observación de los fenómenos tal cual se dieron en su contexto, en cuanto al enfoque fue cualitativo porque explora los fenómenos en profundidad, no tiene secuencia lineal y posee riqueza interpretativa. 
La técnica empleada en la investigación implica un trabajo teórico- práctico. Se manejó la observación etnográfica, luego entrevistas y una encuesta de Wolin \& Woling. con ocho factores especializados.

Para la realización de este trabajo se observó una población de 369 empresarios textiles y confeccionistas del emporio de Gamarra y el tamaño de la muestra fue de 48 empresarios, se calculó por medio de procedimientos estadísticos considerando un error muestral de $5 \%$ y un nivel de confianza del $95 \%$.

Tabla 1.Población y muestra

\begin{tabular}{|c|c|}
\hline \multicolumn{2}{|c|}{ POBLACIÓN DEL ESTUDIO } \\
\hline Población total & 369 \\
\hline Unidad de análisis & $\begin{array}{c}\text { Textiles- } \\
\text { confeccionistas. }\end{array}$ \\
\hline Muestra & 48 \\
\hline
\end{tabular}

Tabla 2. Distribución de la muestra

\begin{tabular}{|l|c|c|c|c|}
\hline & Hombres & Mujeres & Total & $\%$ \\
\hline Textiles & 10 & 11 & 21 & 43.75 \\
\hline Confeccionistas & 12 & 15 & 27 & 56.25 \\
\hline Total & 22 & 26 & 48 & 100.00 \\
\hline
\end{tabular}

Tabla 3. Relación entre variables 


\begin{tabular}{|c|c|}
\hline $\begin{array}{c}\text { VARIABLE } \\
\text { INDEPENDIENTE } \\
\text { LIDERES RESILIENTES } \\
\text { Indicadores }\end{array}$ & $\begin{array}{c}\text { VARIABLE DEPENDIENTE } \\
\text { Indicadores }\end{array}$ \\
\hline - Introspección & Gestión económica \\
- Iniciativa & Eficiencia \\
- Humor & Eficacia \\
- Interacción & Cumplimiento \\
- Independencia & Evaluación \\
- Creatividad & \\
- Moralidad & \\
- Pensamiento critico & \\
\hline
\end{tabular}

\section{RESULTADOS}

En el estudio de la resiliencia se manifiestan las características relevantes de los empresarios de Gamarra, donde el $25 \%$ posee independencia, el $17 \%$ creatividad y el 21 $\%$ iniciativa. Características asociadas directamente a la personalidad resiliente que convierte las adversidades en oportunidades.

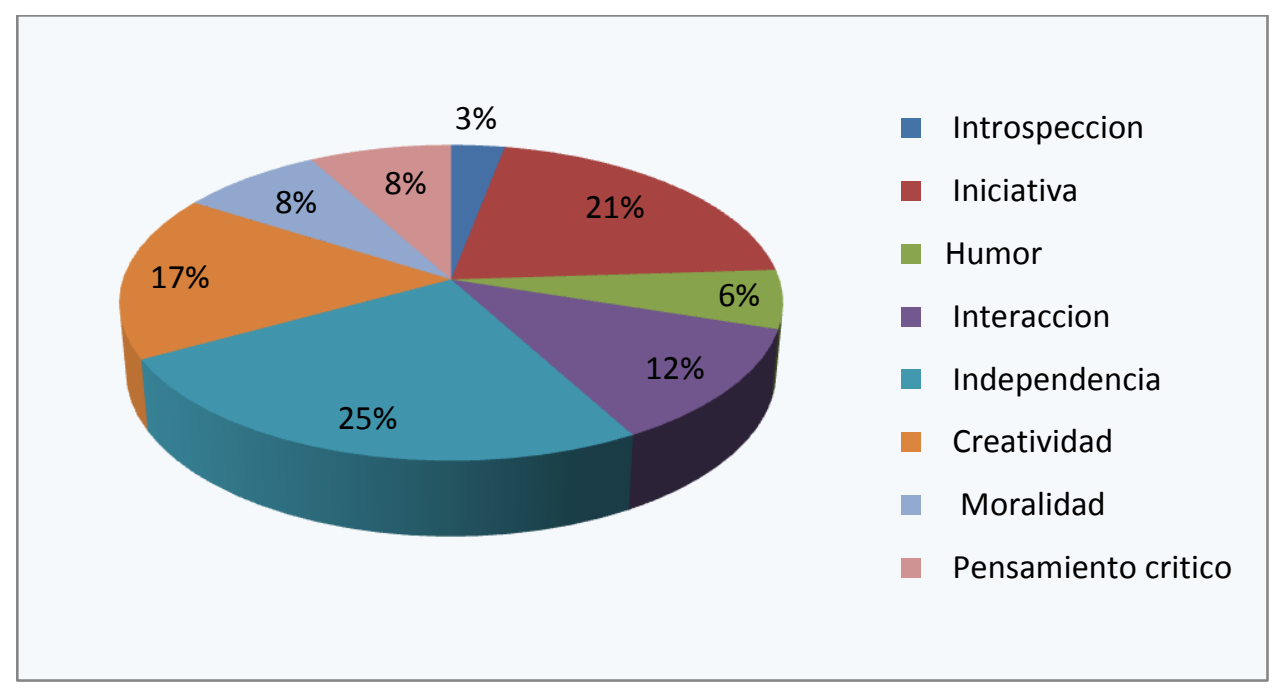

Figura 1. Características de los líderes resilientes.

Respecto a los indicadores de gestión se llega a la conclusión que la eficiencia y eficacia, es decir el uso adecuado de los recursos empresariales y el logro de metas se relacionan 
con las características de los líderes resilientes, ya que estos tienden a trabajar en entornos difíciles y sobreponerse y realizar una gestión económica eficiente.

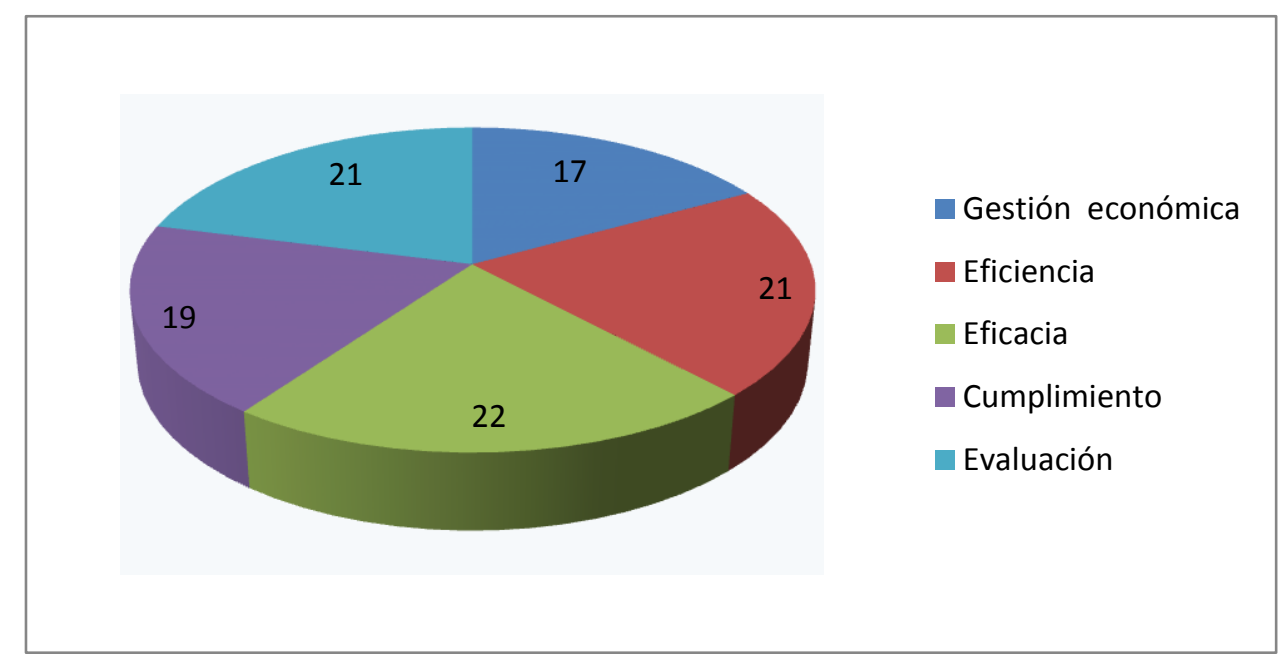

Figura 2. Indicadores de gestión

\begin{tabular}{|c|c|c|c|c|c|}
\hline Estrato & Micro & Pequeña & $\begin{array}{c}\text { Mediana } \\
\text { y } \\
\text { Grande }\end{array}$ & Total & $\%$ \\
\hline$=--\ldots$ Textil & 22,554 & 734 & 103 & 23,391 & $21.0=$ \\
\hline Manufacturera de Madera y Papeles & $\overline{18,874}-$ & $--369-$ & $\overline{-} \overline{6}$ & 19,269 & 17.3 \\
\hline Metal Mecánica & 15,993 & 631 & 47 & 16,671 & 15.0 \\
\hline Agroindustria & 15,504 & 519 & 76 & 16,099 & 14.5 \\
\hline Otras Industrias Manufactureras & 13,836 & 226 & 19 & 14,081 & 12.6 \\
\hline Edición e impresión & 9,016 & 215 & 19 & 9,250 & 8.3 \\
\hline Pieles y Cueros & 4,654 & 161 & 4 & 4,819 & 4.3 \\
\hline Minería No Metalica & 3,405 & 127 & 22 & 3,554 & 3.2 \\
\hline Quimicos & 2,072 & 474 & 56 & 2,602 & 2.3 \\
\hline Joyas y Artículos conexos & 1,095 & 13 & 6 & 1,114 & 1.0 \\
\hline Pesca & 49 & 88 & 32 & 169 & 0.2 \\
\hline Sidero Metalurgía & 123 & 27 & 6 & 156 & 0.1 \\
\hline Instrumento de óptica y relojes & 132 & 3 & 1 & 136 & 0.1 \\
\hline Petróleo y derivados & 27 & 9 & 1 & 37 & 0.0 \\
\hline Total Empresas & 107,334 & 3,596 & 418 & 111,348 & 100.0 \\
\hline \multicolumn{6}{|l|}{$\begin{array}{l}\text { Micro: } 1 \text { a } 10 \text { trabajadores } \\
\text { Pequeña: } 11 \text { a } 100 \text { trabajadores }\end{array}$} \\
\hline
\end{tabular}

Tabla 1. Número de empresas del sector textil en Lima de la micro, 


\section{pequeñas, medianas y grandes.}

\section{CONCLUSIONES}

La resiliencia empresarial está directamente relacionada el liderazgo ya que en las empresas textiles analizadas el cambio organizacional necesario para salir de la crisis fue planeado por un líder, el cual no solo entendía la verdadera problemática de la Mype, sino que a su vez poseía un gran sentido de pertenencia por la organización, el cual nunca le dejo vislumbrar la posibilidad de liquidar la empresa.

La resiliencia organizacional es un estudio que en la actualidad tiene dos condiciones principales, la primera es que las empresas hayan entrado en crisis y la segunda es que estas hayan logrado recuperarse. En el emporio de Gamarra la capacidad de poseer iniciativa es alta y este factor hace posible que los empresarios sujetos de estudio tengan una capacidad resiliente al vencer la adversidad y no darse por vencidos.

\section{BIBLIOGRAFÍA}

- Booz A. H.(2004), "Redefiniendo la agenda del gobierno corporativo. De la administración del riesgo a la resiliencia empresarial",

- Clegg, S. (2007), "Encontrando sentido a la globalización en la teoría de la organización", en S. Porras (coord.), Estudios organizacionales y asuntos globales, México, UAM-Iztapalapa, pp. 69-133.

- Fersen H. León Villamar (2015): “La Resiliencia: su aplicación en el sector empresarial", Revista Contribuciones a la Economía (Febrero 2015). En línea: http://eumed.net/ce/2015/1/resiliencia.html

- López, A. (2009), "El lado positivo de la crisis", CNNexpansión.com, 28 de octubre, en <www. cnnexpansion.com/manufactura/2009/10/28/ el-lado-positivo-de-lacrisis> [consultado el 18 de abril de 2011].

- Marcos, J. y S. Macaulay (2008), “¿Qué es la resiliencia organizacional?”, Nexpansión.com 26 de noviembre, en <www.cnnexpansion. $\mathrm{com} /$ manufactura/especiales/bfque-es-la-resiliencia- organizacional> [consultado el 18 de abril de 2011].

- Marcos, J. y S. Macaulay (2008), “¿Qué es la resiliencia organizacional?", Nexpansión.com 26 de noviembre, en <www.cnnexpansion. $\mathrm{com} /$ manufactura/especiales/bfque-es-la-resiliencia- organizacional> [consultado el 18 de abril de 2011].

- Porras, S. (2007: 227-266), “¿Negocios globales? Redes organizacionales”, en S. Porras (coord.) (2007), Estudios organizacionales y asuntos globales, México, UAM-Iztapalapa. 